\title{
Principais parâmetros biológicos avaliados em erros na fase pré-analítica de laboratórios clínicos: revisão sistemática
}

Primeira submissão em 21/06/11 Última submissão em 10/08/11 Aceito para publicação em 18/03/12 Publicado em 20/06/12

\section{Main biological parameters evaluated in pre-analytical phase errors at clinical laboratories: a systematic review}

Vivaldo Gomes da Costa'; Marcos Lázaro Moreli ${ }^{2}$

\begin{abstract}
unitermos
resumo

Pré-analítico

O diagnóstico laboratorial passou por várias mudanças devido à automatização. Além do aparelhamento, a

Controle de qualidade adoção de um programa de garantia de qualidade (PGQ) está resultando em maior precisão nos exames. Para fins de controle em qualidade, o processamento de uma amostra biológica é didaticamente dividido em Revisão sistemática

Erro laboratorial três fases: pré-analítica, analítica e pós-analítica. Na fase pré-analítica ocorre a maioria dos erros; a divulgação dessas falhas e sua discussão corroboram para a eficiência dos PGQs. No presente estudo, descrevemos as principais variações biológicas encontradas na fase pré-analítica em laboratórios clínicos, por meio de uma revisão sistemática. Fizeram parte dessa revisão 14 artigos. Os parâmetros biológicos descritos na revisão foram, principalmente, glicose, colesterol, triglicérides, enzimas e hormônios. Um erro frequente na punção venosa foi o uso prolongado do torniquete e entre as principais causas de erros observadas foram tempo de armazenamento, tempo de torniquete, técnicas de flebotomia, falta de informações aos pacientes, relação incorreta sangue/anticoagulante, tubos inadequados, amostras contaminadas, medicamentos e alterações interlaboratoriais. Nossos achados são similares aos encontrados em outras pesquisas, mas não encontramos estudos que avaliassem especificamente as alterações na fase pré-analítica decorrentes do uso de medicamentos. Concluímos que os parâmetros biológicos mais avaliados coincidiram com os exames de rotina e uma das formas de evitar às inexatidões laboratoriais é pôr em prática um eficiente PGQ e a produção de trabalhos que explanem o assunto a pacientes e profissionais da saúde.
\end{abstract}

\section{abstract}

Laboratory diagnosis has undergone several changes due to automation. Both equipment supplying and the introduction of quality assurance programs (QAP) have led to higher precision in tests. With the aim to promote quality control, the processing of biological samples comprises three phases: pre-analytical, analytical and post-analytical. Most errors occur in the pre-analytical phase. Thus, their determination and corresponding assessment maximize QAP efficiency. In this study, by means of a systematic review, which comprised 14 articles, we describe the main biological variations found in the pre-analytical phase at clinical laboratories. The biological parameters described in the review included glucose, cholesterol, triglycerides, enzymes and hormones. As far as venipuncture is concerned, a common error was the prolonged use of tourniquet. The main error causes were the following: storage time, tourniquet time, phlebotomy techniques, insufficient information to patients, incorrect blood/anticoagulant ratio, inadequate tubes, contaminated samples, medication and interlaboratory alterations. Our results corroborated other studies, although we did not find other investigations that specifically evaluated changes in the pre-analytical phase due to the use of medication. The most assessed biological parameters coincided with clinical tests. Accordingly, both the implementation of an efficient QAP and the development of professional awareness may prevent laboratory inaccuracies.

\section{key words}

Pre-analytical

Quality control

Systematic review

Laboratory error 


\section{Introdução}

Os laboratórios seguem normas e/ou recomendações que visem diminuir erros ou mesmo evitá-los, sendo existentes erros frequentes que, em grande parte, não alteram significativamente o resultado de um exame. Portanto, é necessário que o profissional da saúde, seja atuando em laboratórios de análises clínicas ou de pesquisas, tenha consciência desses procedimentos e evitem erros o máximo possível para não influenciar diretamente no diagnóstico por meio de resultados falso-positivos e/ou falso-negativos ${ }^{(1,24)}$.

Os programas de garantia de qualidade (PGQ) devem abranger desde a preparação do paciente para a coleta até a liberação dos resultados dos exames, e todo esse processo é dividido em três fases: pré-analítica, analítica e pós-analítica ${ }^{(1,27)}$.

A fase pré-analítica compreende a preparação do paciente, a anamnésia, a coleta e o armazenamento de amostras, ou seja, é a etapa laboratorial que antecede o processamento dos analitos ${ }^{(22,27)}$. Já a fase analítica refere-se à realização do ensaio propriamente dito. No momento, essa etapa é a mais automatizada e para seu controle existem diversos parâmetros avaliados, como precisão, sensibilidade, especificidade, exatidão, entre outros. Ao avaliar esses índices, é preciso estar atento à calibração da aparelhagem, à conservação dos reagentes e ao uso de cálculos matemáticos, como o gráfico controle tipo Levey-Jennings, que analisa a imprecisão de determinado analito(22, 27). Por fim, a fase pós-analítica, etapa final do processo, consiste na obtenção dos resultados, incluindo a interpretação dos ensaios e a caracterização do diagnóstico.

Cada fase é fundamental no laboratório, visto que os erros embutidos na fase inicial, média ou final alterarão o resultado do produto e, consequentemente, sua interpretação(1, 22, 27).

\section{Metodologia}

A revisão sistemática, como qualquer outro estudo, necessita de um projeto de pesquisa. O método deste estudo seguiu as recomendações para a realização de revisões sistemáticas propostas pela Colaboração Cochrane ${ }^{(8)}$.

\section{Amostragem}

A natureza do presente estudo é revisão sistemática. Durante este trabalho, os revisores avaliaram, independentemente, os títulos e os resumos de todos os relatos de ensaios clínicos identificados na busca eletrônica. Portanto, a amostragem foi escolhida por conveniência por meio da concordância da maioria dos revisores.

\section{Fontes de estudos}

As fontes de estudos utilizadas consistiram nas seguintes bases de dados eletrônicas: Literatura Latino-Americana e do Caribe em Ciências da Saúde (LILACS), MEDlars onLINE (MEDLINE - Literatura Internacional em Ciências da Saúde) e Scientific Electronic Library Online (SciELO). Os seguintes descritores foram utilizados para seleção inicial dos artigos: diagnóstico, laboratório, erro laboratorial, fase pré-analítica, controle de qualidade e laboratório clínico. Os trabalhos disponíveis na íntegra, nos idiomas inglês, português e espanhol, publicados no período de 2000 a 2011, constituíram nossa fonte de estudo $(7,8,14)$.

\section{Apresentação dos resultados}

Os estudos incluídos nessa revisão sistemática são demonstrados, resumidamente, na Tabela 1, com dados referentes à sua identificação como os autores, ano de publicação, periódicos e base de dados na seção resultados.

\section{Resultados e discussão}

A pergunta norteadora do presente estudo foi: na literatura científica, quais são os principais parâmetros biológicos analisados em erros na fase pré-analítica de um laboratório clínico? A fase pré-analítica concentra a maioria dos erros laboratoriais e as consequências podem ser determinantes para um diagnóstico falso-positivo e/ou falso-negativo. $\mathrm{Na}$ literatura científica, é fundamentado que esses erros ocorrem por omissão do paciente ou do profissional de saúde ou por falta de conhecimento ${ }^{(28,29)}$.

A revisão sistemática é classificada como um estudo observacional retrospectivo e, dessa forma, permite analisar diversos trabalhos com a mesma temática, mas que diferem em sua metodologia, tendo como consequência a reunião de todos os indícios que possam esclarecer a pergunta norteadora. Em nosso caso, o presente estudo utilizou informações oriundas de diversos artigos e contribuirá na divulgação das fontes de erros frequentes nos laboratórios clínicos. Assim, enfatizamos que essas falhas podem ser evitadas por meio da adoção de um $\mathrm{PGQ}^{(27-29)}$. 
Durante o período de fevereiro a maio de 2011, foram selecionados artigos científicos nos bancos de dados eletrônicos LILACS, MEDLINE e SciELO. Com a seleção de descritores e os critérios de inclusão/exclusão, ocorreu um refinamento para 24 artigos (os demais trabalhos foram excluídos por não atenderem aos objetivos do presente estudo e por estarem disponíveis apenas seus resumos). Finalmente, 14 artigos foram selecionados para constituir a revisão sistemática (Tabela 1).

\section{Principais parâmetros biológicos}

Os analitos são definidos como substâncias na amostra que é determinada por um sistema analítico. A Figura mostra os principais analitos mensurados com erros em sua dosagem, principalmente na fase pré-analítica.

Os parâmetros biológicos alterados que apareceram em maior número nos estudos foram, em ordem decrescente, glicose, colesterol, triglicérides, enzimas, eletrólitos e hormônios. Os resultados evidenciados são similares a alguns estudos encontrados na literatura, por exemplo, a glicose é um analito muito avaliado e sua dosagem é útil para confirmação do diabetes melito, um distúrbio metabólico relacionado com a hiperglicemia, muito comum na população humana ${ }^{(20,22)}$.

No estudo de Menezes et al. ${ }^{(20)}$, foi observado que a glicemia é alterada por diversos fatores, como tempo de

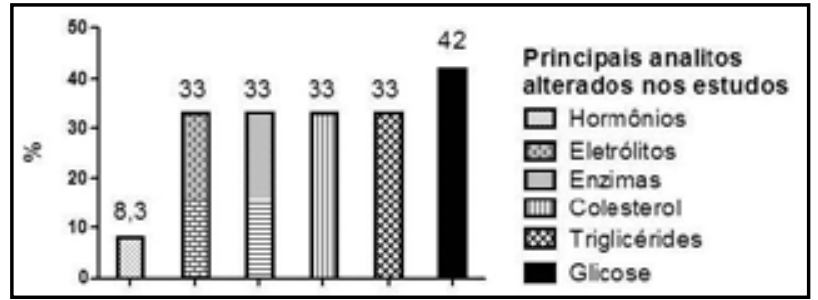

Figura 1 - Analitos comumente citados nos estudos selecionados

Os parâmetros biológicos mais citados nos trabalhos coincidem com os exames de rotina.

armazenamento e velocidade de centrifugação. Nesse estudo experimental, tubos com gel separador, específicos para mensurar a glicemia, foram centrifugados em tempos diferentes - o primeiro tubo foi centrifugado após uma hora da coleta; o segundo, após duas horas; e o terceiro, após três horas. Foi constatado um declive glicêmico considerável quanto maior foi o tempo entre a coleta e a centrifugação da amostra. Houve significativa alteração de $2 \pm 12 \mathrm{mg} / \mathrm{dl}$ de glicose. Essa variação pode ser prejudicial para o paciente, pois ele sai de um estado pré-diabetes para valores aceitos como normal(20,22).

Outro parâmetro biológico constantemente analisado no levantamento bibliográfico foi o colesterol. Nesses artigos, a dosagem de colesterol foi discutida em boa parte dos trabalhos, aparecendo em 33\%. Statland ${ }^{(30)}$ observou um aumento de $5,1 \%$ na mensuração do colesterol quando a oclusão venosa prolongou-se por mais de dois minutos. Guder et al. ${ }^{(12)}$ evidenciaram um aumento de $8,5 \%$ na dosagem do

\section{Tabela 1 Estudos encontrados na literatura científica durante o período de fevereiro a maio de 2011}

\begin{tabular}{|c|c|c|c|}
\hline Autor(es) & Periódico & Base de dados & Ano de publicação \\
\hline Guimarães, A. C. et al. ${ }^{(13)}$ & Rev HCPA & LILACS & 2011 \\
\hline Menezes, E. G. B. et al.(20) & Rev Newslab & SCIELO & 2010 \\
\hline Cabral, E. C. et al. ${ }^{(5)}$ & RBAC & SCIELO & 2010 \\
\hline Goswami, B. et al..(11) & Clin Chem Lab Med & MEDLINE & 2010 \\
\hline Berlitz, F. A. (2) & J Bras Patol Med Lab & LILACS/ScIELO & 2010 \\
\hline Tenor, D. P. et al.(31) & Rev Lab Clin & LILACS & 2010 \\
\hline Lillo, R. et al.(17) & Enferm Clin & LILACS & 2009 \\
\hline Lima-Oliveira, G. S. et al..(18) & J Bras Patol Med Lab & LILACS/ScIELO & 2009 \\
\hline Ferreira, C. E. S.; Andriolo, A. ${ }^{(9)}$ & J Bras Patol Med Lab & LILACS/ScIELO & 2008 \\
\hline Freirea, L. M. D. et al. ${ }^{(10)}$ & RBAC & LILACS/ ScIELO & 2008 \\
\hline Carraro, P.; Plebani, M. ${ }^{(6)}$ & Clinical Chemistry & MEDLINE & 2007 \\
\hline Berlitz, F. A.; Haussen, M. L. ${ }^{(3)}$ & J Bras Patol Med Lab & LILACS & 2005 \\
\hline Vieira, J. G. ${ }^{(32)}$ & Arq Bras Endocrinol Metab & LILACS/ScIELO & 2002 \\
\hline Plebani, M.; Carraro, P.(25) & Clinical Chemistry & MEDLINE & 1997 \\
\hline
\end{tabular}


colesterol quando compararam alterações posturais (pacientes deitados, sentados e em pé) durante a venopunção. É preciso ficar atento a essas alterações, pois coletas em crianças são problemáticas e muitas vezes é necessária sua contenção, porém as recomendações de repouso evitam, em grande parte, as variações ortostáticas da volemia.

Os eletrólitos são comumente avaliados em laboratórios clínicos e são representados principalmente pelos cloretos, potássio, sódio, bicarbonato, magnésio, fosfato e cálcio. São importantes para o equilíbrio da pressão osmótica e a distribuição da água no organismo. Como já comentado, esses íons são facilmente alterados em procedimentos incorretos, como mostrado no estudo de Statland( ${ }^{(30)}$, em que foi verificada uma redução do potássio em 6,2\% quando uma oclusão venosa prolongou-se por mais de dois minutos. Guder et al. ${ }^{(12)}$ também observaram resultado semelhante, porém foram avaliadas as mudanças de postura durante a coleta, como deitado e em pé, e a mensuração do cálcio foi maior que $5 \%$.

Em relação ao tempo de torniquete, Kaplan e Pesce ${ }^{(15)}$ obtiveram uma alteração sérica do potássio de $3,9 \mathrm{mmol} / \mathrm{l}$ para $4,9 \mathrm{mmol} / \mathrm{l}$ referentes ao não uso e à aplicação do torniquete durante dois minutos, respectivamente. Nesse caso, observamos um aumento de $25 \%$ na dosagem desse eletrólito.

As enzimas compreendem um grande grupo de moléculas representadas por alanina aminotransferase (ALT), aspartato aminotransferase (AST), desidrogenase lática (LDH), fosfatases, entre outras. São encontradas alteradas principalmente quando ocorre destruição celular, oclusão de canais, como os ductos biliares, e neoplasias. Statland ${ }^{(30)}$ observou uma variação elevada de 9,3\% em uma oclusão venosa de três minutos. Miller et al. ${ }^{(21)}$ encontraram dados semelhantes, porém o analito com maior variação foi a creatinoquinase, enzima utilizada como marcador bioquímico de lesão miocárdica, durante uma venopunção com oclusão por cerca de seis minutos.

Nas dosagens das enzimas, foram pouco abordados os efeitos da hemólise, quando ocorre degradação das hemácias, quanto ao extravasamento de muitas enzimas para o soro, fazendo que essas proteínas acabem se elevando em relação aos valores normais de referência. A fim de evitar os efeitos da hemólise nas dosagens biológicas, é preciso controlar as variáveis envolvidas nesse processo, como tempo de armazenamento, temperatura e desproporção no sangue/anticoagulante ${ }^{(15,22)}$.

Os triglicérides fazem parte do perfil lipídico e são constantemente quantificados nos laboratórios clínicos.
A alta prevalência dos triglicérides ocasiona turvidez nas amostras e dificulta a leitura em aparelhos automatizados. A determinação dos triglicérides foi realizada por Miller et al.(21) e foi verificada uma elevação de mais de $7 \%$ quando da utilização do torniquete por seis minutos. Os triglicérides apresentam massa molecular elevada e o uso do torniquete excedendo um minuto já pode alterá-los acima dos valores de referência, porém não encontramos trabalhos literários que abordassem a dosagem dos triglicérides com efeito em curto tempo de oclusão venosa ${ }^{(5,22,24)}$.

\section{Causas de erros descritas nos artigos}

As principais alterações que resultaram em erros descritas nas pesquisas foram tempo de armazenamento $(78,6 \%)$, tempo de torniquete $(78,6 \%)$, técnica de flebotomia $(64,3 \%)$, falta de informação aos pacientes $(64,3 \%)$, incorreta relação sangue/anticoagulante (57\%), tubos inadequados (50\%), amostras contaminadas (43\%), medicamentos (29\%) e variações interlaboratoriais $(29 \%)^{(2-4,6,9,10)}$.

A demonstração dos erros laboratoriais varia de local a local e depende de um bom PGQ, que seja constantemente avaliado, e programas de aperfeiçoamento profissional ou cursos de reciclagem, nos quais o laboratorista passa pelas diversas seções laboratoriais. Um PGQ eficiente deve auxiliar na escolha dos métodos, equipamentos, reagentes e pessoal, além de promover a inspeção constante de todas as atividades. Seu objetivo é aumentar a segurança dos resultados e garantir efetivamente um produto final de qualidade ${ }^{(13,19,22,26,27)}$.

Um fator importante e discutido de forma breve nos estudos que fizeram parte dessa revisão foi o efeito dos medicamentos nas dosagens analíticas, sendo que as interações medicamentosas são citadas como causas de diversas variações nos parâmetros bioquímicos avaliados. Não encontramos estudos experimentais e nem revisões que abordassem, especificamente, a medicação como indutor de erros na fase pré-analítica, analítica ou

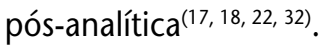

A Tabela 2 mostra resumidamente diversos trabalhos que avaliaram o percentual de erros na etapa inicial de laboratórios clínicos. Podemos observar que a etapa laboratorial com maior concentração de erros foi a fase pré-analítica. Coswami et al. ${ }^{(11)}$ coletaram dados durante um ano e tiveram alto índice de $77 \%$ de falhas nessa área. Os trabalhos de Plebani e Carraro(25) mostraram redução de 68,2\% para $61,9 \%$ no mesmo local de coletas, mas em tempos diferentes. 
Percentagem de erros obtidos na fase

Tabela 2 pré-analítica em diversos estudos

\begin{tabular}{|l|c|c|c|}
\hline Autor & Ano & $\begin{array}{c}\text { Período de } \\
\text { coleta de } \\
\text { dados }\end{array}$ & $\begin{array}{c}\text { Frequência de } \\
\text { erros na fase } \\
\text { pré-analítica }\end{array}$ \\
\hline $\begin{array}{l}\text { Plebani e } \\
\text { Carraro(25) }\end{array}$ & 1997 & 3 meses & $68,2 \%$ \\
\hline $\begin{array}{l}\text { Wiwanitkitt } \\
\text { (33) }\end{array}$ & 2001 & 6 meses & $84,5 \%$ \\
\hline $\begin{array}{l}\text { Carraro e } \\
\text { Plebani(6) }\end{array}$ & 2007 & 3 meses & $61,9 \%$ \\
\hline $\begin{array}{l}\text { Goswami } \\
\text { et al. }{ }^{(11)}\end{array}$ & 2009 & 12 meses & $77,1 \%$ \\
\hline
\end{tabular}

A explicação para os altos índices de falhas nessa etapa laboratorial é a menor automação e também a complexidade dessa etapa de difícil controle; por exemplo, as informações dadas pelos pacientes durante os questionamentos às vésperas da coleta, como omitir se está em jejum, se tomou os medicamentos ou praticou exercícios, são primordiais para garantir a qualidade do exame. Dessa forma, a fase pré-analítica é dependente não apenas do profissional de saúde, mas também da colaboração do paciente ${ }^{(16,23,31,33)}$.

\section{Conclusão}

Os delineamentos de estudos levantados na revisão literária foram revisões narrativas e, principalmente, estudos retrospectivos. Os erros observados durante a punção venosa estão relacionados com tempo elevado na aplicação do torniquete e excesso ou falta de anticoagulante.

Os principais analitos mensurados nos estudos foram glicose, colesterol, triglicérides, enzimas e hormônios e as causas desses erros foram tempo de armazenamento e de torniquete, técnicas de flebotomia, falta de informação aos pacientes, relação incorreta sangue/anticoagulante, tubos inadequados, amostras contaminadas, medicamentos e alterações interlaboratoriais. Uma das medidas adotadas para reduzir ou evitar as inexatidões laboratoriais é a implementação de um eficiente PGQ.

\section{Referências}

1. BANFI, G.; DOLCI, A. Preanalytical phase of sport biochemistry and haematology. J Sports Med Phys Fitness, v. 43, p. 223-30, 2003.

2. BERLITZ, F. A. Controle da qualidade no laboratório clínico: alinhando melhorias de processos, confiabilidade e segurança do paciente. J Bras Patol Med Lab, v. 46, n. 5, p. 353-63, 2010.

3. BERLITZ, F. A.; HAUSSEN, M. L. Seis sigma no laboratório clínico: impacto na gestão de performance analítica dos processos técnicos. J Bras Patol Med Lab, v. 41, n. 5, p. 301-12, 2005.

4. BONINI, P. et al. Errors in laboratory medice. Clin Chem, v. 48, p. 691-98, 2002.

5. CABRAL, E. C. et al. Determinação sérica de lipídeos: variações interlaboratoriais. $R B A C$, v. 42 , n. 2, p. 1158, 2010.

6. CARRARO, P.; PLEBANI, M. Errors in a stat laboratory: types and frequencies 10 years later. Clin Chem, v. 53, n. 7, p. 1338-42, 2007.

7. CASTRO, A. A. Revisão sistemática e metálise. 2001. Disponível em: <http:D:IMyDocumentslald_aulas 1 _ MBEIMBE_3_Principais Desenhos|Revisão Sistemátical atg_meta_analises_3.doc >. Acesso em: 10 mar. 2011.

8. CLARKE, M.; OXMAN, A. D. Cochrane reviewers' handbook 4.1. In: Review manager. The Cochrane collaboration. Oxford, 2000. Disponível em: <http://www.cochrane.dk/ cochrane/hanbook.htm >. Acesso em: 20 maio 2011.

9. FERREIRA, C. E. S.; ANDRIOLO, A. Intervalos de referência no laboratório clínico. J Bras Patol Med Lab, v. 44, n. 1, p. 11-6, 2008.
10. FREIREA, L. M. D. et al. Controle de qualidade laboratorial pré-analítico: avaliação de solicitações médicas de exames bioquímicos no hospital de clínicas da Universidade Estadual de Campinas, São Paulo, Brasil. $R B A C$, v. 40, n. 2, p. 143-5, 2008.

11. GOSWAMI, B. et al. Evaluation of errors in a clinical laboratory: a one-year experience. Clin Chem Lab Med, v. 48, n. 1, p. 63-6, 2010.

12. GUDER, W. G. et al. Samples: from the patient to the laboratory: the impact of preanalitical variables on the quality of laboratory results. Git Verlag GMBH, 1996.

13. GUIMARÃES, A. C. et al. O laboratório clínico e os erros pré-analíticos. Rev HCPA, v. 31, n. 1, 2011.

14. JUSTO, L. P. et al. Revisão sistemática, metanálise e medicina baseada em evidências: considerações conceituais. J Bras Psiquiatr, v. 54, n. 3, p. 242-7, 2005.

15. KAPLAN, L. A.; PESCE, A. J. Clinical chemistry. Theory, analysis, correlation. 3. ed. St. Louis: Mosby, 1996. p. 72.

16. KLOETZEL, K. et al. Controle de qualidade em gestão primária à saúde. A satisfação do usuário. Cad Saúde Pública. Rio de Janeiro, v. 14, n. 3, p. 263-8, 1998.

17. LILLO, R. et al. Variabilidad en los errores preanalíticos del laboratorio entre centros periféricos de extracción: un reto para la seguridad del paciente. Enferm Clin, Alicante, 2009. Disponível em: <http://www.elsevier.es/ sites/default/files/elsevier/eop/S1130-8621(09)001454.pdf>. Acesso em: 14 abr. 2011. 
18. LIMA-OLIVEIRA, G. S. et al. Controle da qualidade na coleta do espécime diagnóstico sanguíneo: iluminando uma fase escura de erros pré-analíticos. J Bras Patol Med Lab, v. 45, n. 6, p. 441-7, 2009.

19. LOPES, H. J. J. Garantia e controle de qualidade no laboratório clínico. Analisa, Belo Horizonte, p. 1-25, 2003. Disponível em: <http://www.goldanalisa.com.br/ publicacoes/Garantia_e_Controle_da_Qualidade_no_ Laboratorio_Clinico.pdf>. Acesso em: 16 maio 2011.

20. MENEZES, E. G. B.; CUNHA, N. C. W.; NETO, R. M. Comparação da determinação da glicose sanguínea usando o tempo como variável. Newslab, Fortaleza, edição 100, 2010. Disponível em: <http://www.newslab. com.br/newslab/revista_digital/100/artigo-1.pdf >. Acesso em: 14 abr. 2011.

21. MILLER, M.; BACHORIK, P. S.; CLOEY, T. A. Normal variation of plasma lipoproteins: postural effects on plasma concentrations of lipids, lipoproteins, and apoliproteins. Clin Lab, v. 38, p. 569-74, 1992.

22. MOTTA, V. T. Bioquímica clínica para o laboratório. 5. ed., Rio de Janeiro: Medbook, 2009.

23. NOVARETTI, M. C. Z. et al. Dez anos de experiência em controle de qualidade em imuno-hematologia. Rev Bras Hematol Hemoter, v. 31, n. 3, p. 160-5, 2009.

24. OLIVEIRA, G. S. L. Estudo de fonte de erros nos processos de flebotomia com ênfase na estase venosa em parâmetros bioquímicos. Curitiba, 2007. Tese (Mestrado) - Ciências Farmacêuticas Análises Clínicas, Universidade Federal do Paraná, 2007.

25. PLEBANI, M.; CARRARO, P. Mistakes in a stat laboratory: types and frequency. Clin Chem, v. 43, n. 8, p. 134851, 1997.
26. PNCQ. Programa Nacional de Controle de Qualidade. Circular nº092/2005 - PNCQ. Disponível em: <http:// www.pncq.org.br/pdfs/rdc_302_rt_lab_cli.pdf>. Acesso em: 10 mar. 2011.

27. POP. Procedimento Operacional Padrão. A importância de se padronizar tarefas nas BPLC. Disponível em: <http://www.anvisa.gov.br/reblas/cursos/qualidade17/ MP\%20_apostila_\%205\%20-\%20final.pdf>. Acesso em: 25 abr. 2011.

28. RIERA, R. et al. Revisões sistemáticas e metanálises na reumatologia. Rev Bras Reumatol, v. 46, p. 8-11, 2006.

29. SAMPAIO, R. F.; MANCINI, M. C. Estudos de revisão sistemática: um guia para síntese criteriosa da evidência científica. Rev Bras Fisioter, v. 11, n. 1, p. 83-9, 2007.

30. STATLAND, B. E. Fundamental issues in clinical chemistry. Am J Pathol, v. 95, p. 243-72, 1979.

31. TENOR, D. P. et al. Aproximación matemática para la corrección de la influencia de la hemólisis en pruebas frecuentes del laboratorio clínico. Rev Lab Clin, Toledo, v. 3, n. 1, 2010. Disponível em: <http://www.elsevier.es/sites/default/files/elsevier/ pdf/282/282v03n01a13146122pdf001 >. Acesso em: 14 maio 2011.

32. VIEIRA, J. G. H. Avaliação dos principais problemas pré-analíticos e metodológicos em dosagens hormonais. Arq Bras Endocrinol Metab, v. 46, n. 1, 2002.

33. WIWANITKIT, V. Types and frequency of preanalytical mistakes in the first. Clin Pathol, v. 1, p. 1-5, 2001. 